\title{
Neuropsychological deficits in depression - a challenge for cognitive-behavioral therapies
}

\author{
Bartosz Piasecki, Karolina Kabzińska* \\ Department of Clinical Psychology, Poznan University of Medical Sciences, Poland \\ * Corresponding Autor: Karolina Kabzińska, Department of Clinical Psychology, Poznan University of Medical \\ Sciences, 70 Bukowska Street,60-812 Poznań, Poland, phone: +48881966933, email: kabzinska.ump@gmail.com \\ a (iD) https://orcid.org/0000-0002-2567-682X \\ b (D) https://orcid.org/0000-0001-5515-8954
}

\begin{abstract}
Neuropsychological deficits in depression are a significant therapeutic challenge. Their occurrence means poor therapeutic prospects, worse social and professional functioning after therapy, as well as a higher risk of relapse. Despite clinical improvement, they often remain even in a state of complete remission. Beck's model of depression does not include interventions directed at neuropsychological processes leading to neurocognitive mechanisms responsible for the development and maintenance of depression. More recent trends in cognitive-behavioral therapy seem to involve neuropsychological processes to a greater extent. This applies to Well's metacognitive model, which focuses on the meta-level of thinking. Therapeutic process involves various aspects of attention, as well as detached mindfulness. Available empirical studies indicate that this therapy model is more effective in reducing neuropsychological deficits than Beck's model. Acceptance and commitment therapy as well as mindfulness-based cognitivetherapy both focus on the development of skills that are related to the efficiency of executive functions and flexibility of attention, i.e. the cognitive processes whose deficits are characteristic of depression. However, research is needed to confirm their effectiveness in reducing neuropsychological deficiencies compared to other therapeutic models. Interventions in the field of cognitive remediationcan be used to enrich cognitive-behavioral therapies and increase their effectiveness. Until now, they have been used as a separate form of therapy, for example in anorexia.
\end{abstract}

Keywords: depression, neuropsychological deficits, cognitive-behavioral therapy.

\section{Introduction}

Depression is a frequent and recurrent disorder associated with a significant deterioration of quality of life and ability to perform social roles, as well as a greater risk of premature death due to suicide [1]. According to the World Health Organization (WHO), the total number of people suffering from depression is 322 million and its incidence is estimated at around 4.4\% [2]. Diagnosis of a depressive episode according to ICD-10 requires the occurrence of at least two of the following symptoms: depressed mood, loss of interest and enjoyment, reduced energy or increased fatigability, as well as at least two consecutive symptoms: decrease in respect for or trust in yourself, excessive and unjustified sense of guilt or irrational remorse, recurrent thoughts of death, suicide or any suicidal behavior, reduced ability to concentrate and think, change in appetite, sleep disorders, changes in psychomotor activ- 
ity. Symptoms must be present for most of the day almost every day for at least two weeks and cause significant suffering or impairment [3].

Neuropsychological deficits are a major challenge in the therapeutic process of depression. Their occurrence is associated with a negative impact on both the immediate results of therapy and the long-term functioning of patients, as well as a higher risk of relapse [4, 5]. Despite the clear overall improvement and positive response to therapy, neuropsychological symptoms often persist [6]. A meta-analysis of research on neuropsychological functioning of patients with recurrent depression showed significant deficits in attention, memory and executive functions [7]. Cognitive deficits have also been observed in patients with depression in remission $[4,8]$. It is indicated that the neuropsychological dysfunctions are often insufficiently diagnosed and therapists focus primarily on the emotional and somatic health aspects and negative patterns of thinking $[8,11]$. The results obtained by observation and self-report on cognitive functioning in depression often differ from neuropsychological tests results $[10,11]$.

\section{Aim}

The aim of this paper is to analyze the existing models of cognitive-behavioral psychotherapy (Beck's classic model, Wells's metacognitive therapy, acceptance and commitment therapy, and mindfulness-based Cognitive Therapy) in terms of their use in work on neuropsychological deficits in depression. In addition, possible directions of development of the analyzed therapies will be considered in the context of their potential for increased effectiveness of depressive patients treatment.

\section{Beck's cognitive model of depression}

The cognitive model of depression proposed by Beck [12-15] is still widely used by therapists. In this model, it is assumed that due to the experience of significant loss in the early stages of life, permanent cognitive structures are formed. The result is a greater vulnerability of the individual to depression in the situation of future losses. Those patterns can remain dormant and be activated by negative life events. Key beliefs and conditional assumptions that arose as a result of early experiences predispose to the occurrence of depressive symptoms in the case when events (e.g. next loss) activate these beliefs and violate hidden assumptions. Negative processes and thinking contents that are triggered lead to the consolidation of depressed mood and other depressive symptoms [12-15]. Beck and Bredemeier theorized that the above-described cognitive mechanism of depression is part of a natural, evolutionary mechanism (the so-called "depression program"), which in specific conditions is adaptive to people and allows the conservation of energy resources. Depressive beliefs play a crucial role of a mediator between information processing, autonomic nervous system, immune response and depressive symptoms. Too long activation of the "depression program" leads to the strengthening of depressive patterns, which increases the risk of relapse [16]. Beck assumed the existence of the so-called cognitive triad of depression consisting of distorted, negative beliefs about yourself, the world and the future. These beliefs usually appear in the present moment in the form of negative automatic thoughts (NATs). They maintain the symptoms of depression, namely they cause a depressed mood and a drop in energy and motivation that lead to a lower engagement in satisfying activities, which becomes evidence of negative beliefs $[12,14,15]$. For people in depression, cognitive distortions are also characteristic. Beck et al. [12] pointed to specific logical errors in patients with depression - primarily generalization, selective attention and dichotomous thinking. These processes increase access to information in accordance with negative beliefs and suppress information that is not consistent with them. This prevents verification of negative cognitive patterns and leads to their maintenance.

Cognitive-behavioral psychotherapy based on Beck's model is always adapted to the specifics of functioning of a given patient, but it is based on certain principles and assumptions. It is a directive-oriented therapy, limited in time, focused on the "here and now", structured and assuming constant cooperation between the therapist and the patient. The basic direction of 
therapeutic work is the identification and reconstruction of cognitive distortions. For this purpose, a number of techniques are used, such as psychoeducation, Socratic dialogue, analysis of arguments for and against, down and up arrows, scaling, relaxation exercises, activation, imaginative exercises and behavioral experiments [13-15]. In therapeutic protocols based on Beck's model, four phases are usually specified in which emphasis is placed on different aspects of the disorder $[12,13,17]$. The first phase of therapy focuses on building a therapeutic relationship, completing conceptualization and defining goals, as well as on the education about the disorder and the cognitive-behavioral model, and finally on the behavioral activation of the patient. The second phase focuses on identifying emotions, negative automatic thoughts, cognitive distortions and then modifying them through the use of appropriate interventions. In the next phase, interventions are increasingly focused on the intermediary assumptions and key beliefs of the patient. The last phase of therapy is a summary and termination of the therapeutic process and prevention of relapse.

Cognitive-behavioral psychotherapy based on Beck's model has repeatedly proved its effectiveness while working with many disorders, including depression $[13,18,19]$. The severity of clinical symptoms of depression often fails to be directly related to the severity of neurocognitive dysfunctions, and full remission of clinical symptoms often does not lead to remission of neuropsychological deficits $[4,5,8,20]$. In Beck's model, there are no interventions that would focus directly on neuropsychological deficits typical of depression and concerning executive functions or attention. Randomized studies conducted by Porter et al. [5] and Groves et al. [9] showed that in patients who participated in cognitive-behavioral psychotherapy based on this model, there were no significant changes in neuropsychological deficits despite significant clinical improvement. Those deficits involved executive functions, memory and verbal learning, as well as processing speed. These results require confirmation in further research, but they show possible limitations in Beck's therapeutic model. An interesting development of Beck's concept aimed at the neuropsychological sphere is the metacognitive model of depression proposed by Wells [21].

\section{The metacognitive model}

Wells's model assumes that the tendency to negative thinking and dysfunctional beliefs are important elements in the development and maintenance of mental disorders, including depression (which is consistent with Beck's model) [21]. Wells concentrates on the repetitive, unhelpful patterns of thinking, beliefs about thinking (metacognitive beliefs), and attentional bias forming the so-called 'cognitive attentional syndrome' (CAS) [21]. The CAS consists of the processes worrying, directing attention to the threat, and the use of unhelpful coping strategies that sustain negative thinking processes. In contrast to Beck's theory, in Wells's model, negative thoughts are considered a trigger factor activating dysfunctional ways of processing or thinking. These, on the other hand, play a key role in developing and sustaining symptoms. The metacognitive model does not focus directly on the content of negative thoughts (e.g. "I am worthless"), but on how the individual processes it (e.g. ruminates or worries about it). The metacognitive model of depression is composed of [21]:

, positive meta-beliefs about the need to ruminate as a way to reduce depressive symptoms or find a way to solve the problem,

, negative meta-beliefs about the inability to control ruminations, the risks associated with depression, and the individual's own vulnerability,

, reduced meta-ruminating awareness,

- CAS (ruminating, focusing on the threat, unhelpful coping strategies).

The metacognitive model of depression was presented in Figure 1.

The two depression therapies based either on the metacognitive model or on Beck's model have a lot in common. In both therapies, patients' activation, increasing their motivation, as well as changing their behavioral patterns that support the disorder are important elements. Also, both are aimed at the reconstruction of negative beliefs using similar therapeutic techniques. However, in the case of metacognitive therapy the focus is on identifying and changing the meta-beliefs, both negative and positive. An important difference in the therapeutic process, especially important in the deliberations on the impact of therapy on neuropsychological deficits, lies in attention 


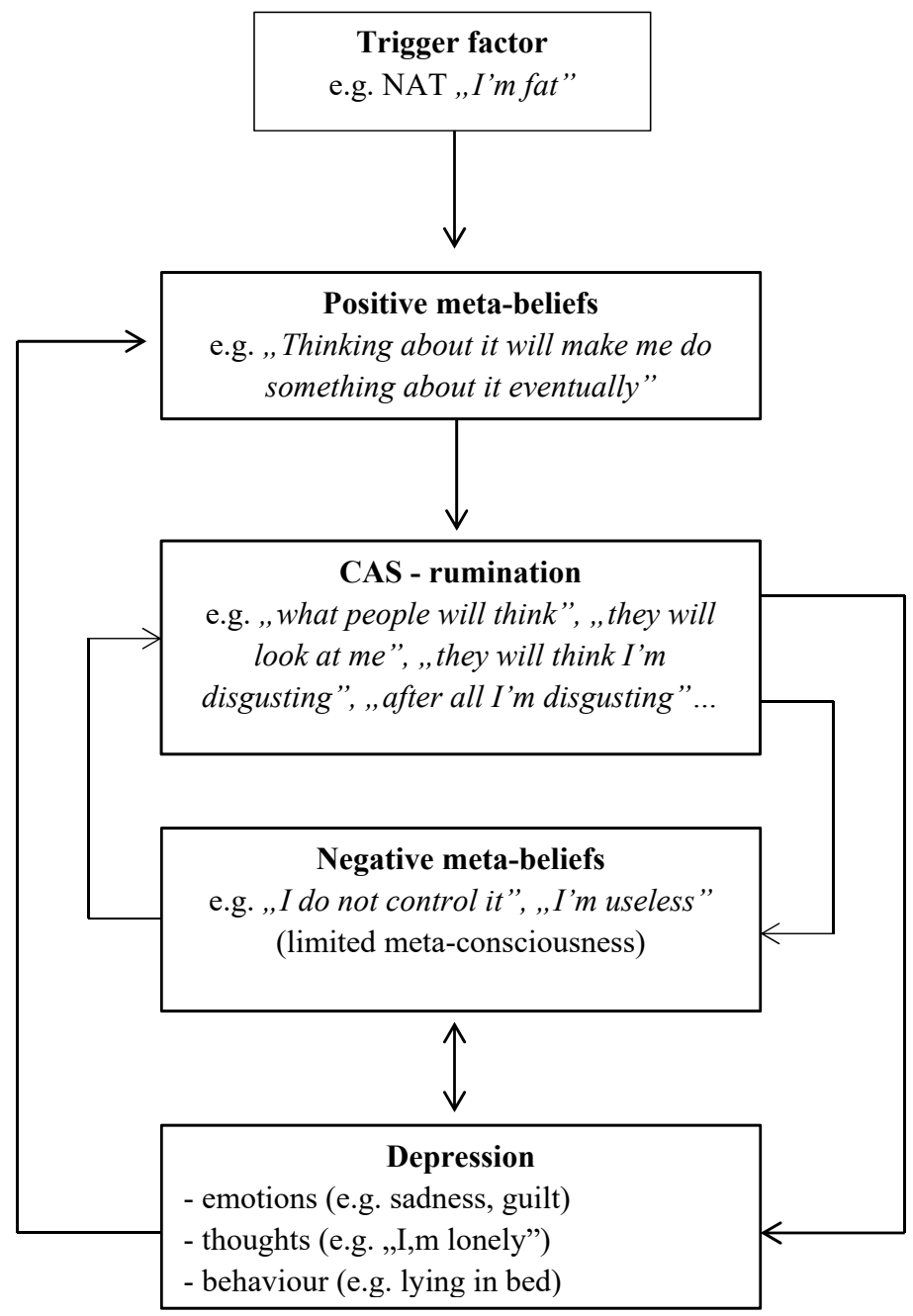

Figure 1. Metacognitive model of depression. Source: based on Wells (2009)

training techniques (ATT) and detached mindfulness, which is the central element of metacognitive therapy [21]. ATT consists of three elements that are practiced during the therapy:

, selective attention (keeping the patient's attention on specific sound stimuli and ignoring others),

, attention switching (shifting attention of the patient to different sound stimuli at an increasing rate),

, divisibility of attention (deepening and maintaining attention to various sound stimuli simultaneously).

The detached mindfulness is understood by Wells (2009) as a state of awareness of internal cognitive experiences (thoughts, memories, beliefs, imaginings, etc.) without engaging in their assessment, control, suppression, change or behavioral response to them. This experience is lived as independent of the whole experience of yourself, from the perspective of the observer. This skill develops during therapy through a series of exercises conducted during the session, as well as in the form of homework [21]. ATT and detached mindfulness are interventions whose aim is to increase self-awareness, minimize perseverative thinking patterns in the form of ruminations, engage in unhelpful behaviors and increase the flexibility of attention. From the neuropsychological perspective, these are elements that can be directly related to the operation of executive functions whose deficits are characteristic of depression. The executive functions are a complex system that supervises, controls and directs the cognitive activity of the individual $[22,23]$. The meta-level of cognitive functioning, which is key in Wells's model, is to a large extent identical with the role of executive functions. ATT and detached mindfulness as an essential element of the therapeutic process 
enable a direct impact on possible deficits in this area of neuropsychological functioning. Previous research seems to confirm this thesis. Metacognitive therapy has been shown to be effective in reducing both clinical symptoms of depression $[24,25]$ and improving neuropsychological deficits [26, 27]. Groves [9] compared in randomized studies the effectiveness of cognitive-behavioral psychotherapy according to both Beck's and Wells's models in reducing neuropsychological deficits. After the end of therapy, the group of patients who participated in Wells's metacognitive therapy was characterized by a significantly greater improvement in the scope of attention and executive functions in comparison with the group in therapy according to Beck's model. The metacognitive model, seems to have an advantage over Beck's model of therapy in improving neuropsychological deficits in patients with depression. According to the author's knowledge, these are the only studies so far comparing the effectiveness of various psychotherapeutic models in terms of changes in neuropsychological functioning in depression and further research in this area is necessary.

\section{Mindfulness-based and accep- tance and commitment therapies}

Elements of metacognitive therapy are also present in approaches belonging to the so-called third wave of cognitive-behavioral psychotherapy, namely mindfulness-based cognitive therapy (MBCT) and acceptance and commitment therapy (ACT). Both approaches are used in psychotherapy of depression.

MBCT for depression is an extension of the stress relief program based on mindfulness created by Jon Kabat-Zinn $[28,29]$. The MBCT program for depression is an eight-week training in the development of mindfulness through the regular and systematic use of meditation in many forms, both at sessions and at home [29, 30]. Mindfulness is understood as a special kind of attention, conscious, non-judgmental and directed at the present moment $[28,29]$. All meditation exercises rely on the learning of a gentle, purposeful focus on the present and appreciating the perception of things as they are. In contrast to the detached mindfulness of Wells's model, in MCBT an atten- tive approach is practiced. It is about directing attention not only to the cognitive sphere but also to the experiences flowing from the body, emotions or the outside world [21]. Mindfulness training allows and individual to develop the ability of conscious, purposeful attention targeting, greater self-awareness of own thoughts and feelings, decentration by getting a distanced observer perspective for own cognitive or affective processes. As a result, the tendency to identify with them and automatically react to them decreases. This allows the individual to reduce inefficient thought processes (in particular ruminating and worrying) and unhelpful prevention strategies that are characteristic of depression [29,30].

The acceptance and commitment therapy is a relatively new approach derived from the cognitive-behavioral trend. The primary goal of ACT is to increase psychological flexibility. This goal is achieved through the use of psychological interventions based on mindfulness (in the same sense as in MCBT) and acceptance combined with strategies of behavior modifications [31, 32]. Psychological flexibility is understood here as the ability to fully, consciously experience the present moment, "here and now", and behave in a way that allows achieving goals consistent with the values of the individual [31, 32]. It consists of:

, contact with the present moment (careful, conscious experience and getting involved in everything that is happening at the moment),

, acceptance (of painful feelings, all other feelings, desires, emotions as they are and making them a place in the field of our experience),

, I as a context (awareness of thoughts, feelings, experiences, actions, conscious observation of them from cognitive distance, taking the perspective of an observer),

, values (desirable characteristics of action, ideals of directing action),

, involved activity (effective action in accordance with professed values),

, cognitive defusion (distancing oneself from one's own cognitive processes, not identifying with them, not engaging automatically in them).

People with depression are characterized by a lack of psychological flexibility. They avoid experiencing negative emotions or thoughts. They are characterized by limited self-awareness and cognitive fusion with negative beliefs (typi- 
cal of depression). In ACT, the immediate goal of working with a depressed patient is not to reduce symptoms, but to release and develop those elements that block the individual's psychological flexibility. Relief of depressive symptoms is treated as a desirable and highly probable side effect of increasing the individual's psychological flexibility [31].

Both ACT and MBCT in their therapeutic interventions focus on cognitive elements that have proved effective in the metacognitive model in working on neuropsychological deficits in depression. These models develop thinking at the meta-cognitive level, increase self-awareness and attentive, flexible and conscious directing of attention. They develop the ability to take a distanced, decentered perspective on experiences, as well as the ability to not react in an automatic manner in response to thoughts, impulses or emotional states that arise in the mind. Skills developed in these therapies are related to the efficiency of executive functions [22, 23]. However, according to the author's knowledge, there are no studies to date focusing on the effectiveness of ACT and MBCT in neuropsychological deficits reduction in depression. A meta-analysis [33] allows to formulate cautious predictions about a possible positive impact on this area also in people with depression - in particular on the selectivity of attention, working memory and executive functions.

\section{Cognitive remediation}

As indicated earlier, the problem of neuropsychological deficits is often overlooked during the therapeutic process, and psychological interventions focus on other areas of patient functioning. Cognitive-behavioral psychotherapy is a model that integrates various techniques and influences after empirically verifying their effectiveness [15]. It is reasonable to look for methods that could increase its effectiveness in working on neuropsychological dysfunctions. An idea worth considering is the introduction of the so-called cognitive remediation (CR) interventions into therapeutic protocols (like using ATT in Well's model). CR showed its effectiveness, among others, in work with schizophrenic patients, elderly patients and brain injuries [5, 22, 34].
CR should be treated as a general name for psychological interventions aimed at working with specific cognitive processes. The choice of methods should therefore be preceded by an appropriate diagnosis of existing neuropsychological deficits. In the light of current studies in depression, there are usually difficulties in the efficiency of executive functions, attention and working memory $[5,7]$. Besides ATT, there are many interventions, both standardized and quality, which can be used to develop these neuropsychological functions [22]. There are also many CR protocols and computerized stimulation packages for neuropsychological functions that still require adequate empirical validation [35]. An example of such a protocol is the CR program for anorexia created by Tachanturia, Davies, Reeder and Wykes [27], whose effectiveness has been empirically confirmed [36]. After some modifications, this program could be adapted to the needs of patients with depression. Particularly noteworthy is the study by Priyamvada et al. [37], who focused on the effectiveness of the CR program in patients with depression lasting for a minimum of 2 years. The authors created a 15-session program for the rehabilitation of cognitive attention and memory in depressive patients, which significantly improved the efficiency of these functions in the study population [37]. Despite the promising results, the study had its limitations. Only 30 patients took part in it and there was no control group, which significantly limits the possibility of generalizing conclusions drawn from the results of the study. This program does not require any specialized hardware or software licenses and could be easily replicated or adapted to existing cognitive-behavioral depression protocols for further validation.

\section{Conclusions}

Neuropsychological deficits in depression are a significant therapeutic challenge. Despite their clinical improvement, they often remain even in a state of complete remission. Their occurrence is associated with poor therapeutic prospects, worse socio-occupational functioning after therapy, and a higher risk of relapse $[4,5,38]$. There is still a marked lack of research on neuropsychological functioning in mood disorders, and in particular on therapeutic (pharmacological and psycho- 
therapeutic) impacts on this sphere of functioning. Beck's model of depression does not include interventions directed to neuropsychological processes, which would be a neuro-cognitive basis for the theorized mechanisms responsible for the development and maintenance of depression. Newer trends of cognitive-behavioral therapy involve more neuropsychological processes. This is especially true for the metacognitive model, which focuses on the meta-level of thinking and includes training of various aspects of attention (ATT), as well as training of detached mindfulness as an integral part of the psychotherapy process. Available empirical studies indicate that this model is more effective in reducing neuropsychological deficits than Beck's model [9]. ACT and MBCT therapies also focus on the development of skills that are related to the efficiency of executive functions and flexibility of attention, i.e. cognitive processes whose deficits are characteristic of depression. However, research is needed to confirm their effectiveness in reducing neuropsychological deficiencies compared to other therapeutic models. Methods that can be used to enrich cognitive-behavioral therapies and increase their effectiveness are interventions in the field of cognitive remediation, which until now have usually been used as a separate form of therapy. The metacognitive model by Wells with his ATT shows that these methods can be a complementary part of the classical psychotherapeutic intervention. However, before new models and therapeutic protocols are created and the availability of empirical research on the effectiveness of neuropsychological deficits therapy is increased, all cognitive-behavioral psychotherapists should pay more attention to this aspect of the patient's functioning. Specialists will be able to get a full picture of the patient's condition. Neuropsychological functioning evaluation using standardized tools is worth considering as a standard when working with depressed people. Future research on treating depression and preventing relapse should be oriented towards the neuropsychological sphere. Improving the cognitive functioning of patients will contribute to optimizing prognosis.

\section{Acknowledgements}

\section{Conflict of interest statement}

The authors declare no conflict of interest.

\section{Funding sources}

There are no sources of funding to declare.

\section{References}

1. Spijker J, Graaf R, Bijl RV, Beekman ATF, Ormel J, Nolen WA. Functional disability and depression in the general population. Results from the Netherlands Mental Health Survey and Incidence Study (NEMESIS). Acta Psychiatr Scand 2004;110:208-14. doi:10.1111/j.16000447.2004.00335.x.

2. WHO. Depression and Other Common Mental Disorders. Global Health Estimates 2017.

3. WHO. The ICD-10 Classification of Mental and Behavioural Disorders. 2009.

4. Bortolato B, Carvalho AF, Mclntyre RS. Cognitive dysfunction in major depressive disorder: a state-of-the-art clinical review. CNS Neurol Disord Drug Targets 2014;13:1804-18.

5. Porter RJ, Bowie CR, Jordan J, Malhi GS. Cognitive remediation as a treatment for major depression: A rationale, review of evidence and recommendations for future research. Aust N Z J Psychiatry 2013;47:1165-75. doi:10.1177/0004867413502090.

6. Semkovska M, Quinlivan L, O'Grady T, Johnson R, Collins A, O'Connor J, et al. Cognitive function following a major depressive episode: a systematic review and meta-analysis. Lancet Psychiatry 2019;6:85161. doi:10.1016/S2215-0366(19)30291-3.

7. Hasselbalch BJ, Knorr U, Kessing LV. Cognitive impairment in the remitted state of unipolar depressive disorder: a systematic review. J Affect Disord 2011;134:20-31. doi:10.1016/j.jad.2010.11.011.

8. Rock PL, Roiser JP, Riedel WJ, Blackwell AD. Cognitive impairment in depression: a systematic review and meta-analysis. Psychol Med 2014;44:2029-40. doi:10.1017/S0033291713002535.

9. Groves SJ, Porter RJ, Jordan J, Knight R, Carter JD, Mclntosh VVW, et al. Changes in neuropsychological function after treatment with metacognitive therapy or cognitive behaviour therapy for depression. Depress Anxiety 2015;32:437-44.

10. Lahr D, Beblo T, Hartje W. Cognitive performance and subjective complaints before and after remission of major depression. Cognit Neuropsychiatry 2007;12:25-45. doi:10.1080/13546800600714791.

11. Rohling ML, Green P, Allen LM $3^{\text {rd }}$, Iverson GL. Depressive symptoms and neurocognitive test scores in patients passing symptom validity tests. Arch Clin Neuropsychol Off J Natl Acad Neuropsychol 2002;17:205-22.

12. Beck AT, Rush AJ, Shaw BF, Emery G. Cognitive therapy of depression. New York: Guilford Press; 1979.

13. Beck JS. Cognitive-behavior therapy. New York: Guilford Press; 2011.

14. Fennell M, Bennett-Levy J, Westbrook D. Depresja. Oksfordzki Podręcznik Eksp. Behawioralnych W Ter. Poznawczej, Gdynia: Alliance Press; 2004.

15. Popiel A, Pragłowska E. Psychoterapia poznawczo-behawioralna. Warszawa: Wydawnictwo Paradygmat.; 2008.

16. Beck AT, Bredemeier K. A Unified Model of Depression Integrating Clinical, Cognitive, Biological, and 
(C) 2019 by the author(s). This is an open access article distributed under the terms and conditions of the Creative Commons Attribution (CC BY-NC) licencse. Published by Poznan University of Medical Sciences

Evolutionary Perspectives. Clin Psychol Sci 2016;4: 596-619.

17. Dobson KS. Cognitive Therapy for Depression. Cogn. Ther. Depress. Manag. Complex. Comorbidity, New York: Guilford Press; 2008, p. 3-33.

18. Driessen E, Hollon SD. Cognitive behavioral therapy for mood disorders: efficacy, moderators and mediators. Psychiatr Clin North Am 2010;33:537-55. doi:10.1016/j.psc.2010.04.005.

19. Hofmann SG, Asnaani A, Vonk IJJ, Sawyer AT, Fang A. The Efficacy of Cognitive Behavioral Therapy: A Review of Meta-analyses. Cogn Ther Res 2012;36:427-40. doi:10.1007/s10608-012-9476-1.

20. Bora E, Harrison BJ, Yucel M, Pantelis C. Cognitive impairment in euthymic major depressive disorder: a meta-analysis. Psychol Med 2013;43:2017-26. doi:10.1017/S0033291712002085.

21. Wells A. Metacognitive therapy for anxiety and depression. New York: Guilford Press; 2009.

22. Pąchalska M. Rehabilitacja Neuropsychologiczna. Lublin: Wydawnictwo Uniwersytetu Marii Curie-Skłodowskiej; 2008.

23. Pąchalska M. Neuropsychologia kliniczna. vol. 1. Warszawa: Wydawnictwo Naukowe PWN; 2007.

24. Dammen T, Papageorgiou C, Wells A. An open trial of group metacognitive therapy for depression in Norway. Nord J Psychiatry 2015;69:126-31. doi:10.3109/0 8039488.2014.936502.

25. Jordan J, Carter JD, Mclntosh VVW, Fernando K, Frampton CMA, Porter RJ, et al. Metacognitive therapy versus cognitive behavioural therapy for depression: a randomized pilot study. Aust N Z J Psychiatry 2014;48:932-43. doi:10.1177/0004867414533015.

26. Siegle GJ, Ghinassi F, Thase ME. Neurobehavioral Therapies in the $21^{\text {st }}$ Century: Summary of an Emerging Field and an Extended Example of Cognitive Control Training for Depression. Cogn Ther Res 2007;31:235-262.

27. Tchanturia K, Giombini L, Leppanen J, Kinnaird E. Evidence for Cognitive Remediation Therapy in Young People with Anorexia Nervosa: Systematic Review and Meta-analysis of the Literature. Eur Eat Disord Rev J Eat Disord Assoc 2017;25:227-36. doi:10.1002/ erv.2522.
28. Kabat-Zinn J. Życie, piękna katastrofa. Warszawa: Wydawnictwo Czarna Owca; 2013.

29. Segal ZV, Williams MG, Teasdale JD. Mindfulness-Based Cognitive Therapy for Depression. New York: Guilford Press; 2013.

30. Teasdale JD, Williams M, Segal Z. Praktyka uważności. Ośmiotygodniowy program ćwiczeń pozwalający uwolnić się od depresji i napięcia emocjonalnego. Kraków: WUJ; 2016.

31. Harris R. Zrozumieć ACT. Terapia akceptacji i zaangażowania w praktyce. Sopot: GWP; 2018.

32. Hayes SC, Strosahl KD, Wilson KG. Acceptance and Commitment Therapy An Experiential Approach to Behavior Change. New York: Guilford Press; 2003.

33. Chiesa A, Calati R, Serretti A. Does mindfulness training improve cognitive abilities? A systematic review of neuropsychological findings. Clin Psychol Rev 2011;31:449-64. doi:10.1016/j.cpr.2010.11.003.

34. McGurk SR, Twamley EW, Sitzer DI, McHugo GJ, Mueser KT. A meta-analysis of cognitive remediation in schizophrenia. Am J Psychiatry 2007;164:1791-802. doi:10.1176/appi.ajp.2007.07060906.

35. Bowie CR, Gupta M, Holshausen K, Jokic R, Best $M$, Milev R. Cognitive remediation for treatment-resistant depression: effects on cognition and functioning and the role of online homework. J Nerv Ment Dis 2013;201:680-5. doi:10.1097/ NMD.0b013e31829c5030.

36. Leppanen J, Adamson J, Tchanturia K. Impact of Cognitive Remediation Therapy on Neurocognitive Processing in Anorexia Nervosa. Front Psychiatry 2018;9:96. doi:10.3389/fpsyt.2018.00096.

37. Priyamvada R, Ranjan R, Chaudhury S. Cognitive rehabilitation of attention and memory in depression. Ind Psychiatry J 2015;24:48-53. doi:10.4103/09726748.160932

38. Woo YS, Rosenblat JD, Kakar R, Bahk W-M, Mclntyre RS. Cognitive Deficits as a Mediator of Poor Occupational Function in Remitted Major Depressive Disorder Patients. Clin Psychopharmacol Neurosci Off Sci J Korean Coll Neuropsychopharmacol 2016;14:1-16. doi:10.9758/cpn.2016.14.1.1.

Acceptance for editing: 2019-11-09 Acceptance for publication: 2019-12-30 